\title{
FRAMEWORK, IMPLEMENTATION AND ALGORITHM For ASYNCHRONOUS POWER SAVING OF UWB- Mac With SteErable Directional ANTENNA IN MANETS
}

\author{
Murtala Muhammad $^{1}$, AbubakarGidadoHalilu $^{2}$ and Sanusi Mohammed Bunu ${ }^{3}$ \\ ${ }^{1}$ Department of Computer Science, Adamawa State University, Mubi, Adamawa State, \\ Nigeria. \\ ${ }^{2}$ School of Computing, Science and Engineering, University of Salford, Manchester. \\ M5 4WT \\ ${ }^{3}$ Department of Computer Science, Adamawa State Polytechnic, Yola, Adamawa State, \\ Nigeria.
}

\begin{abstract}
Asynchronous Power Saving (APS) technique is one of the unique standard used in Mobile Ad hoc Networks to conserve more energy in the system. This technique when combined with other power saving systems like Ultra WideBand System and the use of Directional antenna in MANETs provides astonishing result. In designing Medium Access Control (MAC), developing a framework and giving a detailed implementation procedure are among the factors that hinder the process; specifically in MANETs not to mention the Algorithm. This paper aimed at exploring a standard framework, implementation procedure and Algorithm for designing an Asynchronous Power Saving Ultra WideBandMeduim Access Control (UWB-MAC) in MANETs using directional antenna. An implementation procedure that comprises of transmission, channelization, and receiver pattern of the Physical layer is specified in this research work. The paper concludes with an algorithm for an Asynchronous Power Saving UWB-MAC using a Steerable Directional antenna in MANETs.
\end{abstract}

\section{KEYWORDS}

MANETs, UWB-MAC, Asynchronous Power Saving, Steerable Directional Antenna

\section{INTRODUCTION}

The adaptation of Asynchronous Power Saving (APS) techniques in transmission and reception in the design and implementation of Ultra WideBand-Medium Access Control (UWB-MAC) in Mobile Ad hoc Networks (MANETs) is because, the techniqueis more realistic than other standards and also enable long operation time for battery powered devices[1]. This is achievable through providing framework, implementation and detailed algorithm that will ease the design procedure. The APS standard uses an effective method to extend battery life specifically at the MAC layer and enable devices to turn off completely or reduce power for long periods of time[2]. The specified period of time for the standard to extend battery life is relative to the superframe duration. With the aid of Ultra Wideband system combined with the APS technique, the proposed MAC protocol is capable of saving enormous in MANETs[3]. During the transmission or reception period, APS standard offers two different power management modes for the devices to operate on: active and hibernation modes. The active mode transmits and receives beacons in every superframe in the communication medium, while hibernation mode is capable of hibernating multiple superframes that come as a result of cross communication, and 
therefore halts the communication process by dropping the transmitting or receiving frames in those superframes[4]. Subsequently, the technique stretches its capability to support devices that sleep for a particular portion of a superframewhich leads to maximum power saving by the protocol. The aim of this paper is to present a detailed framework, implementation procedures and proposed algorithm of a UWB-MAC using APS techniques in MANETS while utilising directional antenna system.

In this paper, the use of directional antenna as a unique contribution, this have been achieved by specifying the direction of transmission based on a specific angle of arrival, neighbours are coordinated by indicating the intention to hibernate and each device includes a hibernating mode in its beacon. The reason for the hibernating mode is to specify the number of superframes in process[4]. This technique will save enormous amounts of energy by putting non-active devices in sleep mode and thus, will not allow devices to send or receive beacons or any other frames. The operation mode of the proposed asynchronous power saving model is to operate efficiently only if the time spent in that model is greater than a certain threshold.

The major advantage of using the APS model in the implementation of the proposed UWB-MAC is to reduce contention in the communication medium due to the fact that nodes wake up at different time instances[5]. Another benefit attached to using the APS model is that it allows each node in the communication environment to set its own sleep/wake schedule independently[6]. Finally, using the APS model will support the devices to be in Asynchronous mode, meaning they do not have to be synchronised together all the time. At the later end, after presenting the framework of the proposed protocol and the implementation procedure, the algorithm for the implementation of the APS for the proposed UWB-MAC with a steerable directional antenna system will also be presented.

The paper is organised as follows after introduction as section one. Section two of this paper present the framework of the proposed concept and is among requirement that are captured in the title of the paper after implementation and algorithm as major keywords. Section three is the transmission implementation aspect of the physical layer followed by channel and modulation procedures in section four. The receiver part is section five while section six is the multibandOFDM implementation. The main objective of the paper which is the Asynchronous Power Saving UWB-MAC with directional algorithm is presented in section seven. And finally, section eight concludes the paper before the references.

\section{Framework Of The Proposed Aps Uwb-MaC}

Media Access Control (MAC) protocols have different frameworks that varies depending on types, structures and implementations[7]. This paper managed to come up with a proposed framework of the protocol is based on the OSI model architecture from the application layer to the physical layer. The application layer is assumed to refer to multimedia transmission over a multi-hop network and is characterised by sensing activities, channel coding improvement and time varying channel conditions as presented in Figure 1. The transport layer of the proposed protocol is UDP. The test of heavy multimedia applications like VoIP is assumed for testing the viability of the protocol. The network layer signified the use of multi-hop connections with multiple devices to express the nature of MANETs and MB-OFDM support. This also signifies that there exists a communication link between all the devices in the network, all of which are mutually independent. That means each node is capable of laying packets for other nodes in the network with a traverse of multiple nodes in order access a destination device. 


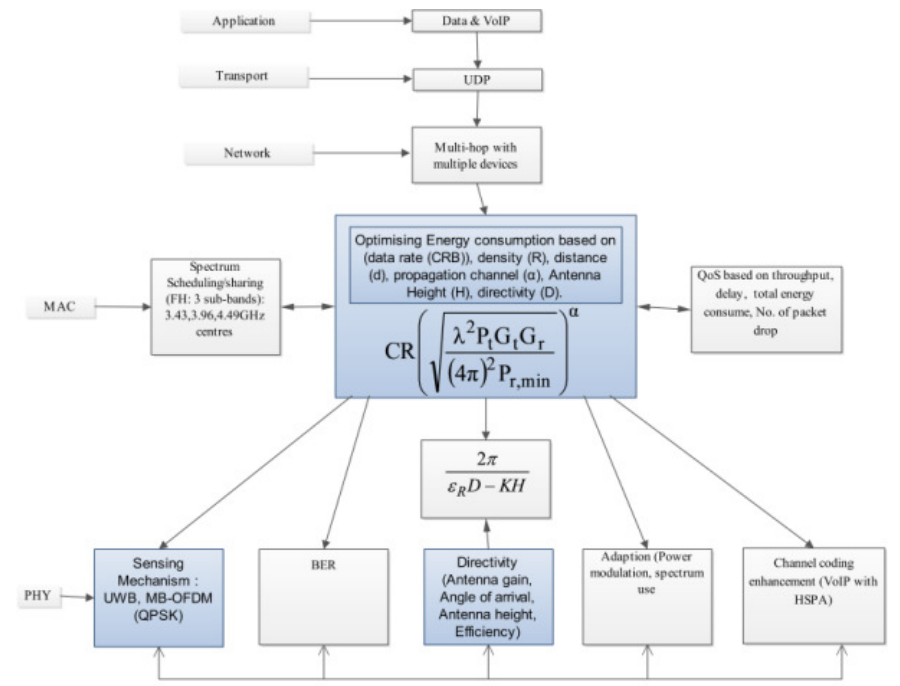

Figure 1: APS Framework of the Proposed UWB-MAC

The implementation of the MAC layer, based on the conceptual framework indicated in Figure 1, is obtained by defining three basic mediums: the spectrum scheduling/sharing, energy optimisation entities and the quality of service of the proposed UWB-MAC. The spectrum scheduling/sharing technique is Frequency-Hopping (FH) with 3 sub-bands of 3.43, 3.96 and $4.49 \mathrm{GHz}$ centres. Testing these three sub-bands for performance precision is beyond the research paper because[8] has covered such a gap. The suitable task done by the FH is to help select the best code for executing sub-channels within the channel and to perform encoding/decoding frames of data. Transmission and reception are synchronised to the hopping sequence and assumed to involve fast hopping which changes the frequency several times in order to transmit a single bit.

The second entity of the proposed UWB-MAC, is energy optimisation which can be iterated after the spectrum scheduling of the frequency-hopping. These are data rate, density of the network, distances between the nodes, propagation channel, Antenna Height and directivity. The behaviour of the proposed system relies on these entities to determine the output of the system. The quality of the proposed protocol based on network quality of service (QoS) parameters such as throughput, delay, total energy consumed at the MAC layer and total numbers of packet drop in the communication medium have been measured. The output varies with the changes in respect to entities of the antenna used and the direction of transmission.

Generally, the PHY layer is responsible for frequency generation, frequency selection, modulation, signal detection and data encryption[9]. However, this paper focused mainly on the sensing strength of the MIMO channel to determine how MB-OFDM performs and the capacity of QPSK performance with respect to changes in the measured entities at the MAC layer. This determines the digital modulation used,Analog-to-Digital Converter (ADC/DAC) resolution, and the internal resolution of the First Fourier Transformation (FFT) algorithm. These are the factors on which the power management depends at the PHY stack of the protocol. In addition, it also determines the data rate of the system, transmitting power, receiving power and deep sleep power of the devices participating in the system although, the performance of the sensing aspect of the PHY stack is beyond the content of this research study. Other sections of the PHY stack are Bit Error Rate (BER), directivity, adaption and channel coding enhance. BER determines the existence of the MIMO system for multi-system transmission and it also serves as a link between the antenna directivity. At the PHY layer, the research focused on the directivity entity as the measure concerned with determining the output of the system. Directivity depends on the antenna 
gain, angle of arrival, antenna height and the efficiency of the antenna. These will determine the power consumed at the PHY stack with respect to modulation and spectrum usage.

Aside from ordinary data packets, VoIP is used as the targeted multimedia application to test the viability of the proposed UWB-MAC. VoIP is typically a very heavy application and requires the enhancement of channel coding to obtain an optimised version of the protocol. Thispaper considered the use of High Speed Packet Access (HSPA) in order to improve download and upload speeds of the voice communication within the PHY stack. The impact of HSPA transmission over the UWB communication channel will stabilise the protocol because of the Band 3 usage, thus allowing an additional noise to the channel by the repeater at the UWB working frequencies.

\section{TRANSMiSSION IMPLEMENTATION OF ASP UWB-MAC}

The implementation of the transmitter section, this study considered the Wireless Sensor Network standard module which is also based on the PHY properties of the 368 standardneeds to be considered. In this case, a complete communication system is modelled, based on the proposed model using a MATLAB Simulink that include elements of transmitter and receiver components array. The transmission section includes an initiating transmitter, transmitting spectrum and input/output transmitter array as presented in Figure 2. Additionally, the system was modelled using Simulink in order to integrate the proposed model to mimic a real PHY layer of the system. The transmitting section generates the input signal through the channel model that alternates the signal noise and passes it to the receiving array and to the beamformer for directivity.

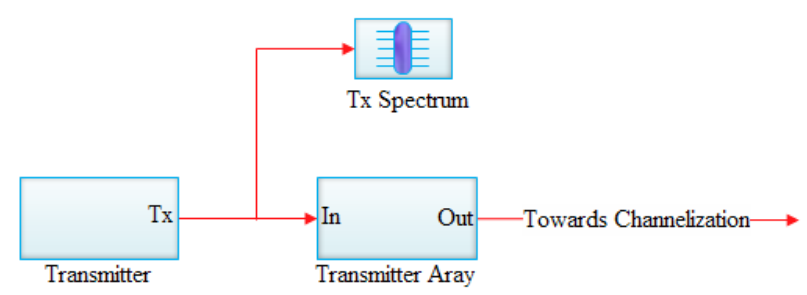

Figure 2: Transmission Path of ASP UWB-MAC

While running the simulation, the desired direction of the transmission could be changed or reset to $22^{0}$ to yield low power in the medium. When the receiving angle is changed or reset to a different degree, for example $90^{\circ}$, the power of the receiving angle increases, thus compensating for the power between $22^{\circ}$ to $90^{\circ}$. When the receiving signal direction is set to $22^{\circ}$, the same as with the transmitting direction, the power also decreases. Subsequently, this integrates the channel and modulation techniques applied to the transmitter in order to forward a proper signal to the receiver.

\section{Channel And Modulation Techniques ApPlied To The TRANSMITTERS}

The channel and modulation techniques applied to the proposed model supports the UWB system with high frequency and maximum data support through the channel for proper implementation of the proposed UWB-MAC. Thepaper adapts the MIMO channel system in the implementation of the proposed model and combines the two possible techniques in the MIMO system that is, multiplexing and beamforming. The reason for integrating both techniques is that multiplexing 
increases the effective data rate by sending parallel beams of data on different antennas at the same time[10], while the beamforming technique focuses on particular narrow angle/signal beams in the direction of the receiver, enhancing the signal reliability[10]. The streams of multiplexing techniques in the MIMO system are represented by line of transmission patterns from the upstream to the downstream (in MANET from DEVt to DEVr). The channel is placed in-between the transmitting and the receiving ends which co-ordinate more of an angle of arrival and beamforming. Before arriving at the beamformer from the transmission channel, a UWB interfering signal is added to the desired signal. When running the simulation, changes can be made on the independent direction from which both the desired and interfering signals are received.

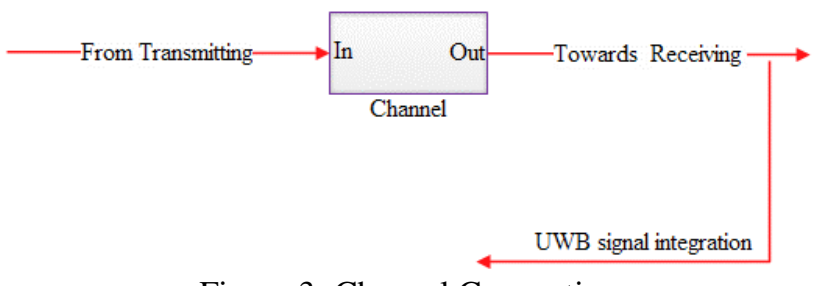

Figure 3: Channel Connection

The UWB channel controls the wide band signal monitors the signal and arrival direction of the signal by combining the concept of beamforming and multiplexing. Figure 4 presents the UWB section of the PHY stack.

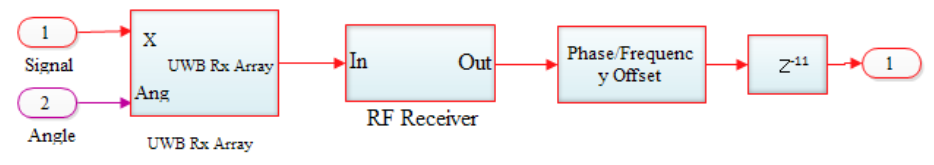

Figure 4: UWB Channel

The mathematical formulation of the MIMO channel has been used to increase the data rate and possibly transmit several information streams in parallel where a special multiplexing technique is applied. The transmitting and receiving sections of the channel can be presented in a vector spaces formulation and later converted into matrices.

$x_{1}$
$\mathrm{~T}_{\mathrm{x}}$ can be $\stackrel{x_{2}}{\vdots}$ and is the $\mathrm{T}$ transmit sample across the channel and is also $\mathrm{T}$-dimensional vector.

$x_{t}$
$y_{1}$
$\mathrm{R}_{2}$ can be
$\vdots$
$y_{r}$ and is the Receiving sample across the channel and is also R-dimensional vector.

Therefore, the two vectors can be represented as an input/output system through the channel to represent the entire wireless channel as in:

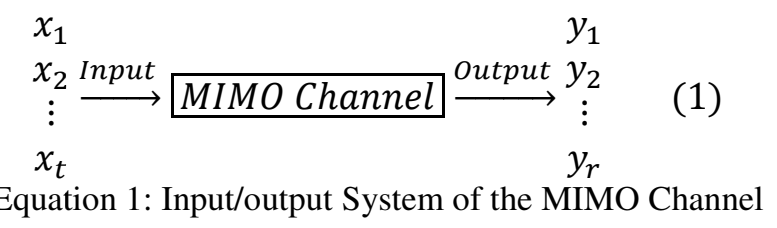

The T-dimensional vector can be represented by $\bar{x}$ and R-dimensional can be represented by $\bar{y}$. We can pass the input to the wireless channel which is the $T_{x}$ vector through the MIMO channel to the output of the wireless system $\mathrm{R}_{\mathrm{x}}$ vector $(\bar{x} \dot{\Rightarrow} M I M O \Rightarrow \bar{y})$. Therefore, the MIMO channel transforms the $\mathrm{T}$-dimensional vector into R-dimensional and this can be technically converted 
into a matrix. The matrix takes an input as T-dimension and the output to be R-dimension vector. Equation 2 is the resulting equation of the channel model can be:

$$
\bar{y}_{r \times 1}=\bar{H} x_{t \times 1}+\bar{w}_{r \times 1}
$$

Equation 2: Resulting Equation of the Channel Model

Where $\bar{w}_{r x 1}$ is the noise of the channel which is also informed of receiving a signal. $\mathrm{H}$ can be represented as $\mathrm{r} x \mathrm{t}$ dimensional vector with a channel coefficient of:

$$
H=\left[\begin{array}{cccc}
h_{11} & h_{12} & \cdots & h_{1 t} \\
h_{12} & h_{22} & \cdots & h_{2 t} \\
\vdots & \vdots & \cdots & \vdots \\
h_{r 1} & h_{r 2} & \cdots & h_{r t}
\end{array}\right]
$$

Equation 3: Channel Coefficient of the Channel

The r-rows are the receiving signal while the t-columns are the transmitting signal. The coefficient of $\mathrm{H}$ as in $\mathrm{h}_{\mathrm{i}, \mathrm{j}}\left(\mathrm{i}, \mathrm{j}^{\text {th }}\right)$ coefficient which means $\mathrm{i}^{\text {th }}$ row and $\mathrm{j}^{\text {th }}$ column are the channel coefficient that represents transmitting antennas of the DEVs in the wireless network. Therefore, $\mathrm{h}_{\mathrm{i}, \mathrm{j}}$ is nothing but the channel coefficient between $\mathrm{i}^{\text {th }}$ receiving antenna and $\mathrm{j}^{\text {th }}$ transmitting antenna. The total number of channel coefficients in the wireless system is the product of receiving and transmitting signals $(\mathrm{r} * \mathrm{t})$. For example, $\mathrm{h}_{3,2}$ is the channel coefficient of $3^{\text {rd }}$ receiving DEV and $2^{\text {nd }}$ transmitting DEV.

\section{RECEIVER IMPLEMENTATION OF ASP UWB-MAC}

The receiver section uses a feedback loop and the block comprises an estimated angle of reception and beamforming algorithms that assumes the two signals are received. The root multiple signal personification (Music) algorithms are used as adapted in[11]. The block estimates two directions and each are processed to the control logic to decide the angle that provides the best modulation regression. The estimated angle is passed to another block of direction of arrival tool box that performs minimum value distortion less response. The block preserves the signal power at the given direction which supresses interference noise coming from other direction. The beamforming angle determines the use of the control logic and the signal strength used to determine which of the two ways of estimated angle provides the lower modulation regression. The particular beamforming algorithm provides excellent tracking of the desired signal even when the interference signal is stronger than the expected signal.

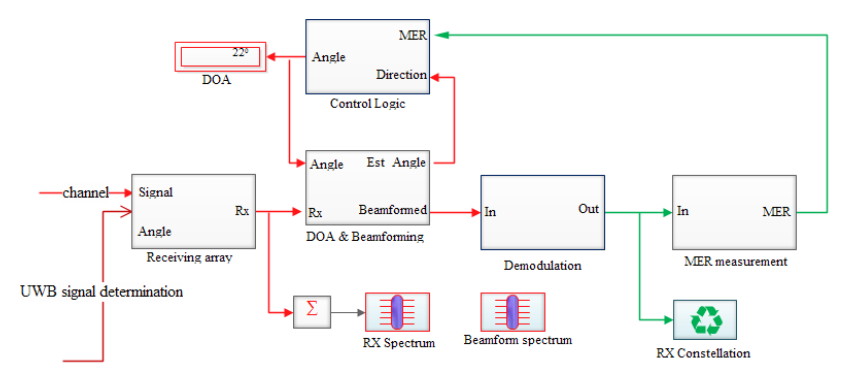

Figure 5: Reception Path of the proposed ASP UWB-MAC

Considering the receiving signal spectrum, the effect of non-linear behaviour occurs due to high power when the angle of transmission is wide. Therefore, from all indications, the direction of arrival has an impact on the system, especially when the direction is wide, and this shows that the proposed model performs better. A correlation of the estimated direction of arrival is made along 
with the spectrum of the receiving signal after beamforming to the constellation of the resulting receive signal when changing the direction of arrival. The receiver recalculates a real weight of the signal in order to maximise the modulation.

The complete PHY layer of the ASP UWB-MAC is the combination of the three basic designs: transmitting, channel and receiving sections. Figure 6 presents the complete diagram of the PHY layer.

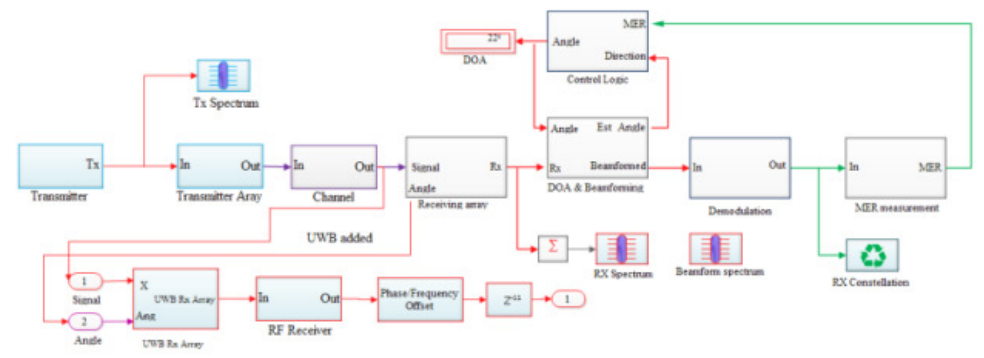

Figure 6: Complete PHY Stack of the ASP UWB-MAC

It is reasonable to elaborate further on the channel utilisation process and the reasons behind adopting MB-OFDM in the design process rather than other techniques. Section 6 elaborates more on the MIMO channel (MB-OFDM channel).

\section{Multiband-OfDM IMPlementation ON ThE Mimo Configuration}

The choice of MB-OFDM for the implementation of the proposed UWB-MAC is based on its robust nature towards multi-path communication[12]. It is easier to implement in terms of spectrum sculpting and the technique is less prone to interference in relation to other technologies like narrowband systems[10]. Narrowband communication systems do not accommodate resilience with regards to high interference within their operational bandwidth limits. Series of data can be modulated using a Gaussian monocycle pulse train, as stated in [13]. It implies using pulse position modulation (PPM).

Basically, Figure 5.7 describes the MB-OFDM Physical layer with all the numbers of groups in the channel. The groups have $528 \mathrm{MHz}$ bands in four distinct groups.

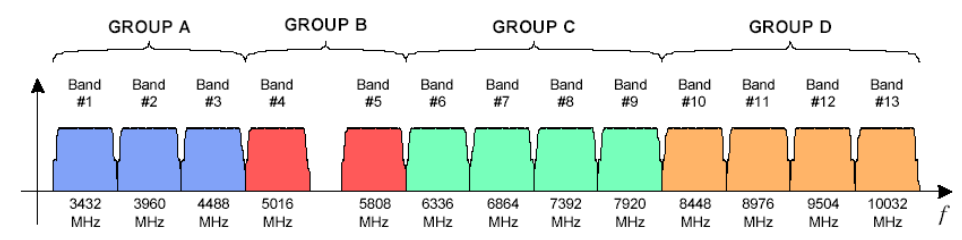

Figure 7: Multi-Band OFDM Physical Layer[14]

The groups are as follows:

i. Group A: is destined for the $1^{\text {st }}$ generation devices and the frequencies range from 3.1 4.9GHz.

ii. Group B: is reserved for future use and the frequencies range from $4.9-6.0 \mathrm{GHz}$.

iii. Group C: is intended for devices with improved SOP performance with frequencies range from $6.0-8.1 \mathrm{GHz}$.

iv. Group D: is also reserved for future use with frequencies range from $8.1-10.6 \mathrm{GHz}$. 
Another consideration that prompted the use of the multi-band approach over other techniques like DS-UWB, is its high spectral efficiency inherent in OFDM and its high resilience to RF interference to other RF signals. Apart from robustness to multi-path effect by MB-OFDM as mentioned earlier, the standard is a proven technology used in an indoor environment like other wireless MAC standards for both PHY and MAC layers.

In Multi-Band OFDM (MB-OFDM), the approach divides the available UWB frequency spectrum of the channel $(3.1 \mathrm{GHz}$ to $10.6 \mathrm{GHz})$ into multiple, smaller paths and no overlapping bands with bandwidths greater than 500MHz. The standard is similar to the narrowband frequency-hopping techniques, with more advantage of eluding transmission over certain bands.

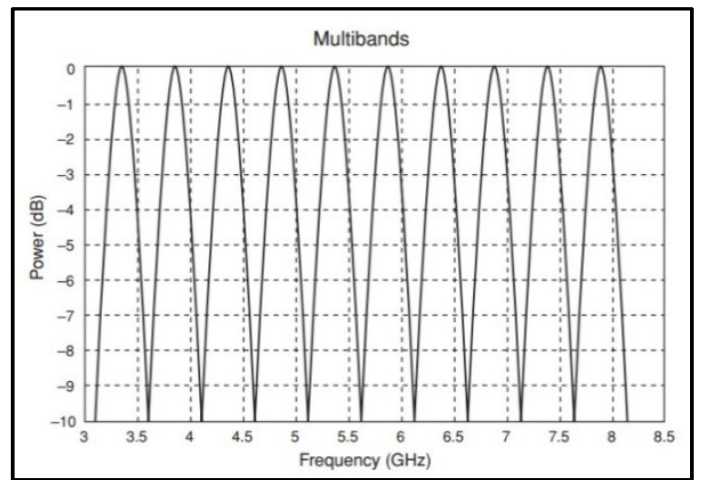

Figure 8: OFDM Modulation Pattern

The study revealed a maximum of $7.5 \mathrm{GHz}$ for UWB 5 channels and channels $1-4$ have 3 subbands each while channel 5 has 2 sub-bands. In the channels, each sub-band occupied 528MHz. The OFDM symbol of the channel has 128 sub-carries and can transmit each symbol for one time slot per channel. The concept of cyclic prefixing has been used to reduce the complexity of the reception $\left(\mathrm{R}_{\mathrm{x}}\right)$ circuitry with multi-path resistance from a prefix of $60 \mathrm{~ns}$ and the use of simple multiplication operations in place of Shift-Multiply-Add in frequency domain although strong consideration has not been placed on the time frame for each and every channel to switch between which is the Guard Interval (GI) and Closed Proximity (CP). However, the default of 9.5ns GI to switch between channels in the communication path has to be considered. To avoid experiencing high latency and redundancy in the proposed UWB-MAC, average GI and CP values have been used and to refrain from complicated channel switching of hardware circuitry, the use of short GI has been avoided. Part of the targeted aim in the proposed UWB-MAC is to decrease high overhead and accumulation of multi-path signal strength. Thus, larger CP adoption was not pursued, rather, an adoption of a CP duration of direct proportion to the delay spread in the channel.

The implementation of the MB-OFDM component of the proposed system involved MATLAB coding functions and graphical results are found by showing the Bit Error Rate (BER) of the system. The BER of the MB-OFDM for the entire system has been determined by plotting a Signal to Noise Ratio (SNR) as shown in Figure 9. To achieve the alleged accurate localisation through the physical layer, BER is controlled in terms of declining to yield maximum throughput. Meanwhile, SNR is also controlled to yield maximum throughput. Moreover, these factors (BER and SNR) are major sources for energy inefficiency in MANET. 


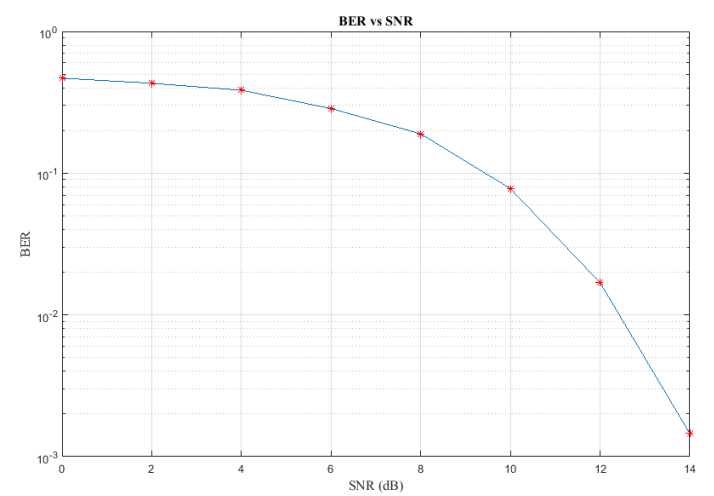

Figure 9: MB-OFDM BER vs SNR Implementation

The complete PHY stack is linked to the UWB-MAC using the Asynchronous Power Saving (APS) model for implementation to save energy in the communication setup.

\section{Asynchronous Power SAVing Algorithm For Uwb-Mac With STEERABLE Directional ANTENNA}

The proposed UWB-MAC protocol algorithm is designed to ensure that, after issuing the RTS command in the transmission process, no member of the extended beacon group transmits a beacon frame at the same time as the device in order to avoid collision. Thus, in line with the algorithm steps, information included in beacon frames is designed to facilitate contention-free frame exchanges by ensuring that a device does not transmit frames while a neighbour is transmitting or receiving frames. To permit correct frame reception, the UWB-MAC protocol algorithm attempts to ensure that a device(s) is/are unique within the device's extended beacon group and direction of transmission is specified otherwise the process will reinitiate for a certain number of times before terminating the process.

The strategy for the implementation of the proposed UWB-MAC with directional antenna based on the APS model is to enable all neighbours in the communication environment to have an overlapping time between their wake periods: the wake period constitutes a preamble and data frame in order to save more energy in the medium. The scheme has a transmitting and receiving mode as in below.

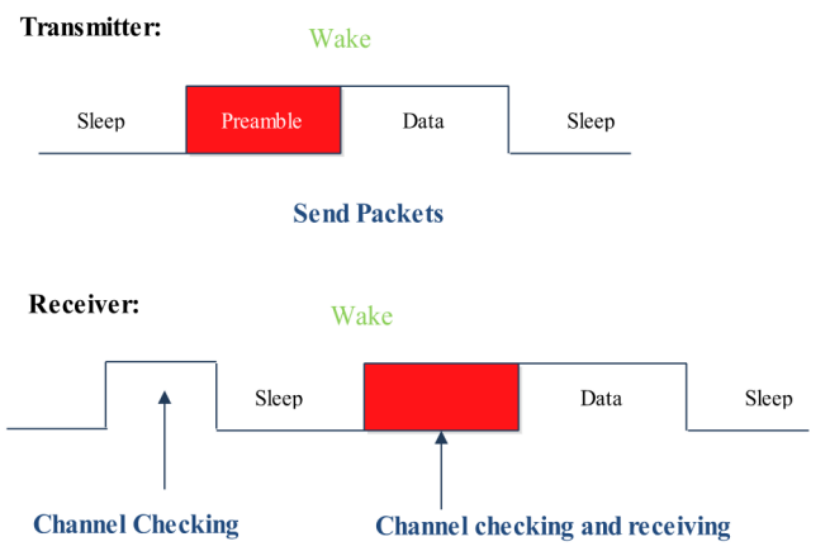

Figure 10: Asynchronous Scheme of the Proposed UWB-MAC

\subsection{Main Algorithm for Proposed APS UWB-MAC}


Item i): outline the transmitting algorithm and item ii): outline the receiving algorithm for the proposed UWB-MAC with steerable directional antenna system. The receiving mode has two additional checking modes for the protocol that is, checking the channel before transmitting and channel checking with receiving mode.

\section{(i) Transmitting and channel determination algorithm}

1. Procedure Algorithm ( ) UWB-MACpConSer Algorithm

2. Time $=$ simulationTime do

3. using a directional RTS packet to transmit

4. is there a valid neighbour(s) within angleOfTransmission? and angleLessThan $360^{\circ}$

5. If $\left(\mathrm{PT}_{\mathrm{x}}>=\min \mathrm{x}\right) \mathrm{OR}\left(\mathrm{PR}_{\mathrm{x}}>=\mathrm{MinR}_{\mathrm{x}}\right)$ then

6. Transmit data $\rightarrow$ yes

7. else

8. Initiate another transmission process with different angle $<360^{\circ}$

9. Is there any blockage or hidden node(s)?

10. If yes then

11. Hibernate non-active devices by putting them on sleep mode

12. Put the channel in idle direction mode

13. If No then

14. Perform line 7 and go to line 3

15. Else

16. Is the channel free to transmit?

17. If yes

18. Transmit with full UWB channel capacity

19. Else

20. Put the channel to idle sensing mode

21. Successful transmission

\section{ii. Receiving and channel determination algorithm}

1. Procedure Algorithm ( ) UWB-MACpConSer Algorithm

2. Time $=$ simulationTime do

3. using a omni-directional CTS packet to transmit

4. is there a valid neighbour(s) within angleOfReception? and angleLessThanOrEqual $360^{\circ}$

5. If $\left(\mathrm{PT}_{\mathrm{x}}>=\min \mathrm{x}\right) \mathrm{OR}\left(\mathrm{PR}_{\mathrm{x}}>=\mathrm{MinR}_{\mathrm{x}}\right)$ then

6. Transmit data $\rightarrow$ yes

7. else

8. Initiate another transmission process with different angle $=<360^{\circ}$

9. Is there any blockage or hidden node(s)?

10. If yes then

11. Hibernate non-active devices by putting them on sleep mode

12. Put the channel in idle direction mode

13. If No then

14. Perform line 7 and goto line 3

15. Else

16. Is the channel free to transmit?

17. If yes

18. Transmit with full UWB channel capacity

19. Else

20. Put the channel to idle sensing mode

21. Successful transmission 


\section{Conclusion}

This paper discussed a clear framework, implementation procedures and algorithm for designing an Ultra Wideband medium access control protocol using an asynchronous power saving techniques. The paper also expresses the importance of using directional antenna system which can also aid in conserving energy in the design and implementation of MAC protocol in MANETs. The method is clearly stated with backup from literatures with detailed diagrams and mathematical expressions. The future work of the research study is to key in the said algorithm in a supported simulator and to link the physical layer of the protocol with the simulator in order to produce results that will support the argument. Network Simulator-3 (ns-3) is chosen for the implementation because of its robustness in terms of supporting different standards and algorithms.

\section{REFERENCES}

[1] K. M. Thotahewa, J. Y. Khan, and M. R. Yuce, "Power efficient ultra wide band based wireless body area networks with narrowband feedback path," IEEE Transactions on Mobile Computing, vol. 13, no. 8, pp. 1829-1842, 2014.

[2] V. K. Sachan, S. A. Imam, and M. Beg, "Energy-efficient communication methods in wireless sensor networks: A critical review," International Journal of Computer Applications, vol. 39, no. 17, 2012.

[3] V. Sharma and R. Kumar, "Cooperative frameworks and network models for flying ad hoc networks: a survey," Concurrency and Computation: Practice and Experience, vol. 29, no. 4, p. e3931, 2017.

[4] D. Triantafyllopoulou, K. Moessner, M. Alam, A. Radwan, and J. Rodriguez, "Cognitive Means Smart: Knowledge Saves Power," in Energy Efficient Smart Phones for $5 G$ Networks: Springer, 2015, pp. 141-166.

[5] M. U. Anyaegbu, "Clock synchronisation for UWB and DECT communication networks," HeriotWatt University, 2013.

[6] J. A. Stankovic and T. He, "Energy management in sensor networks," Phil. Trans. R. Soc. A, vol. 370, no. 1958, pp. 52-67, 2012.

[7] P. Bahl, R. Chandra, T. Moscibroda, G. S. H. Narlanka, Y. Wu, and Y. Yuan, "Media access control (MAC) protocol for cognitive wireless networks," ed: Google Patents, 2014.

[8] E. A. Jorswieck, L. Badia, T. Fahldieck, E. Karipidis, and J. Luo, "Spectrum sharing improves the network efficiency for cellular operators," IEEE Communications Magazine, vol. 52, no. 3, pp. 129$136,2014$.

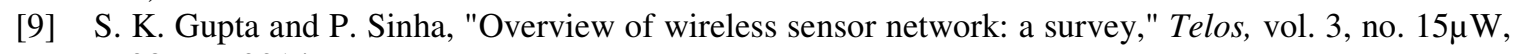
p. $38 \mathrm{~mW}, 2014$.

[10] S. Sun, T. S. Rappaport, R. W. Heath, A. Nix, and S. Rangan, "MIMO for millimeter-wave wireless communications: Beamforming, spatial multiplexing, or both?," IEEE Communications Magazine, vol. 52, no. 12, pp. 110-121, 2014.

[11] S. Varade and K. Kulat, "BER comparison of Rayleigh fading, Rician fading and AWGN channel using chaotic communication based MIMO-OFDM system," International Journal of Soft Computing and Engineering, vol. 1, no. 6, 2012.

[12] A. E. M. HAMZAH, "DISTRIBUTED MULTI-HOP RESERVATION SCHEME FOR WIRELESS PERSONAL AREA ULTRA-WIDEBAND NETWORKS," 2018.

[13] X. Xu, T. Xia, A. Venkatachalam, and D. Huston, "Development of high-speed ultrawideband ground-penetrating radar for rebar detection," Journal of Engineering Mechanics, vol. 139, no. 3, pp. 272-285, 2012.

[14] T. H. Nguyen, T. H. Nguyen, V. T. Tran, and B. G. T. Vu, "A scheme of dual carrier modulation with soft-decoding for MB-OFDM MIMO systems," in Advanced Technologies for Communications (ATC), 2011 International Conference on, 2011, pp. 220-223: IEEE. 